\title{
PATOGENICIDADE CAUSADA PELO FUNGO Colletotrichum gloeosporioides (Penz) EM DIFERENTES ESPÉCIES FRUTÍFERAS ${ }^{1}$
}

\author{
KATIANE SANTIAGO SILVA², TIYOKO NAIR HOJO REBOUÇAS ${ }^{3}$, ODAIR LACERDA LEMOS ${ }^{4}$, MARINÊS PEREIRA \\ BOMFIM $^{4}$, ALEXANDRE ALVES BOMFIM ${ }^{4}$, GREGÓRIO LUNA ESQUIVEL ${ }^{5}$, ANA PAULA PRADO BARRETO ${ }^{4}$, \\ ABEL REBOUÇAS SÃO JOSÉ ${ }^{3}$, NILMA OLIVEIRA DIAS ${ }^{2}$, GILTEMBERGUE MACEDO TAVARES ${ }^{2}$
}

RESUMO - O presente trabalho teve como objetivo avaliar a patogenicidade de Colletotrichum gloeosporioides em frutos de manga, mamão, goiaba e maracujá. A inoculação do C. gloeosporioides foi feita de forma direta, fazendo um furo no fruto e colocando, em seguida, um disco de meio de cultura contendo o micélio do fungo das diferentes espécies. O delineamento experimental utilizado foi inteiramente casualizado, com 25 tratamentos, 3 repetições, sendo as avaliações realizadas por esquema fatorial de 5 x 5 . Os resultados obtidos demonstram que todos os isolados apresentam patogenicidade em todas as espécies frutíferas consideradas. A goiaba é o hospedeiro que apresenta menor suscetibilidade aos diversos isolados de C. gloeosporioides. As mangas Tommy Atkins e Rosa e o mamão apresentam o maior crescimento das lesões, portanto maior suscetibilidade.

Termos para indexação: antracnose, manga, goiaba, maracujá, mamão.

\section{PATHOGENICITY CAUSED BY Colletotrichum gloeosporioides (Penz) IN DIFFERENT FRUITFUL SPECIES}

\begin{abstract}
The present work had as objective to evaluate the pathogenicity of $C$. gloeosporioides isolated from mango, papaya, guava and passion fruit. The inoculation with $C$. gloeosporioides was through a direct way, opening a hole on the fruit and inoculating PDA disks with a mycelium of the fungus. The control was inoculated with PDA disks without the fungus. A completely randomized blocks design was used, with 25 treatments and 3 repetitions. A factorial scheme $(5 \times 5)$ was used for evaluations. The results obtained demonstrate that all the isolated show pathogenicity in all fruit species studied. Guava is the host, which showed smaller susceptibility to the several isolated of $C$. gloeosporioides. Tommy Atkins and Rosa Mangoes and also papaya present larger lesions growth, therefore a larger susceptibility.
\end{abstract}

Index terms: anthracnose, mango, guava, passion fruit, papaya.

As moléstias de plantas são responsáveis por grandes perdas nas culturas de importância econômica, dentre as quais se destacam as doenças de pós-colheita em frutíferas. A perda pós-colheita de frutos tropicais no Brasil situa-se na ordem de $30 \%$ dos produtos comercializados (Tavares, 2004).

Os fungos do gênero Colletotrichum são fitopatógenos importantes nas regiões tropicais e subtropicais do mundo. Esses fungos são os causadores de uma diversidade de doenças como antracnose, podridão de pedúnculo, varicela em manga, abacate e mamão (Bailey \& Jeger, 1992).

A antracnose causada por espécies de Colletotrichum é a principal doença de frutos em pós-colheita, sendo considerada doença de elevada importância econômica no Nordeste do Brasil (Serra \& Silva, 2004). O sintoma típico da doença é caracterizado por lesões arredondadas, grandes, necróticas, com o centro dos tecidos deprimidos, onde são produzidas massas de conídios de coloração alaranjada (Bailey et al., 1992), podendo ocorrer uma podridão-mole nos frutos, prejudicando a sua comercialização (Lima Filho et al., 2003).

O presente trabalho teve como objetivo avaliar a agressividade de Colletotrichum gloeosporioides em frutos de manga, mamão, goiaba e maracujá na pós-colheita.

Os trabalhos foram realizados na Biofábrica de Microrganismos da Universidade Estadual do Sudoeste da Bahia câmpus de Vitória da Conquista, Bahia.

Foram obtidos isolados a partir de lesões de frutos de goiaba (Psidium guajava L.) var. Paluma, mamão (Carica papaya L.) var. Sunrise Solo, maracujá-amarelo (Passiflora edulis f. flavicarpa Deg) e manga (Mangifera indica L.) var. Tommy Atkins, naturalmente infectados, adquiridos em pomares comerciais da região Sudoeste da Bahia. Os frutos foram mantidos à temperatura de $25^{\circ} \mathrm{C}$ para desenvolvimento das lesões da antracnose. Após o desenvolvimento das lesões, procedeuse o isolamento em meio de cultura batata dextrose ágar (BDA) (Goes, 1995). Para a instalação do experimento, frutos de goiaba (var. Paluma), manga (var. Tommy Atkins e Rosa), mamão (var. Sunrise Solo) e maracujáamarelo foram colhidos em pomares comerciais nos municípios de Tanhaçu-BA, Eunápolis-BA, Anagé-BA e Glaucilândia-MG, em ponto de maturação fisiológica utilizado para exportação. Foi feito o tratamento hidrotérmico dos frutos e a desinfecção em solução de hipoclorito de sódio (1\%). As inoculações foram feitas de forma direta, colocando-se um disco do meio de cultura contendo o micélio do fungo $C$. gloeosporioides. Após a inoculação, os frutos foram colocados em câmara úmida por 24 horas. Após esse período, os frutos foram mantidos em temperatura ambiente para a avaliação da severidade da doença que foi realizada a cada 48 horas, medindo-se as lesões em dois sentidos diametralmente opostos. As avaliações foram encerradas quando a maioria dos frutos se encontravam totalmente tomados pelas lesões. $\mathrm{O}$ delineamento experimental adotado foi o inteiramente casualizado, com 25 tratamentos e 3 repetições. As avaliações foram realizadas por esquema fatorial $5 \times 5$. As médias foram comparadas pelo teste de Tukey, a $5 \%$ de probabilidade.

Dos isolados inoculados nos frutos de manga Rosa e Tommy, observa-se na Tabela 1 que aqueles oriundos de manga e goiaba promoveram maior crescimento de lesões sem se diferenciarem entre si. Estes resultados diferem dos obtidos por Peres et al. (2002), quando em testes de patogenicidade constataram que isolados de Colletotrichum gloeosporioides foram mais agressivos em seus hospedeiros de origem com exceção da manga e do maracujá. Nos frutos de mamão e maracujá, houve diferença estatística dos tratamentos somente em relação à testemunha. Serra \& Silva (2004), em testes realizados com isolados de diversas frutíferas, observaram que, em frutos de maracujá, isolados de

${ }^{1}$ (Trabalho 140/05). Recebido: 05/09/2005. Aceito para publicação: 23/02/2006

${ }^{2}$ Eng $^{\mathrm{a}}$ Agra. , M.Sc., Universidade Estadual do Sudoeste da Bahia, Estrada do Bem Querer, Km 04, Caixa Postal 95, CEP 45083-900, Vitória da Conquista-BA, Fone: (77) 3425-9351. E-mail: kasantiago@ig.com.br

${ }^{3}$ Eng Agr. D. Sc., Universidade Estadual do Sudoeste da Bahia, Estrada do Bem Querer, Km 04, Caixa Postal 95, CEP 45083-900, Vitória da Conquista-BA, Fone: (77) 3425-9351. E-mail: tiyoko@uesb.br

${ }^{4}$ Mestrando, Universidade Estadual do Sudoeste da Bahia, Estrada do Bem Querer, Km 04, Caixa Postal 95, CEP 45083-900, Vitória da Conquista-BA, Fone: (77) 3425-9350. E-mail: mestrado.agronomia@uesb.br

${ }_{5}^{5}$ Doutorando, Colégio de Postgraduados, Texcoco, México. E-mail: gluna@ colpos.mx 
TABELA 1 - Médias de crescimento de lesões $(\mathrm{cm})$ causadas por Colletotrichum gloeosporioides em frutos de manga, mamão, maracujá e goiaba após 12 dias de incubação. Vitória da Conquista-BA, 2005.

\begin{tabular}{lccccc}
\hline \multirow{2}{*}{ Isolado } & \multicolumn{5}{c}{ Hospedeiro } \\
\cline { 2 - 5 } & \multicolumn{2}{c}{ Manga } & & \\
\cline { 2 - 5 } & Rosa & $\begin{array}{c}\text { Tommy } \\
\text { Atkins }\end{array}$ & & & \\
\hline Manga & $4,62 \mathrm{a}^{*}$ & $4,99 \mathrm{a}$ & $1,28 \mathrm{a}$ & $3,71 \mathrm{a}$ & $2,84 \mathrm{a}$ \\
Maracujá & $2,54 \mathrm{~b}$ & $1,65 \mathrm{~b}$ & $0,45 \mathrm{a}$ & $3,33 \mathrm{a}$ & $1,68 \mathrm{ab}$ \\
Goiaba & $3,50 \mathrm{ab}$ & $3,93 \mathrm{a}$ & $1,16 \mathrm{a}$ & $2,83 \mathrm{a}$ & $2,17 \mathrm{a}$ \\
Mamão & $1,74 \mathrm{bc}$ & $1,75 \mathrm{~b}$ & $1,03 \mathrm{a}$ & $3,48 \mathrm{a}$ & $2,22 \mathrm{a}$ \\
Testemunha & $0,00 \mathrm{c}$ & $0,00 \mathrm{~b}$ & $0,00 \mathrm{a}$ & $0,00 \mathrm{~b}$ & $0,00 \mathrm{~b}$ \\
\hline
\end{tabular}

Médias seguidas de mesma letra na coluna não diferem entre si, pelo teste de Tukey, a $5 \%$ de probabilidade.

manga e goiaba, induziram sintomas típicos da antracnose, o que concorda com os dados observados neste estudo. Entretanto, segundo os autores, isolado de maracujá não causou lesão no próprio hospedeiro, o que está em desacordo com as observações do presente estudo.

Na Figura 1, são apresentados os resultados da inoculação de C. gloeosporioides proveniente de frutos de manga (var. Tommy Atkins) nos diferentes frutos. Todas as variedades estudadas foram suscetíveis ao patógeno, porém o crescimento das lesões em frutos das duas variedades de manga e nos frutos de mamão foi estatisticamente superior ao crescimento das lesões em frutos de goiaba.

Quando se utilizaram isolados provenientes de lesões em frutos de maracujá (Figura 2), pôde-se observar lento desenvolvimento do fungo inoculado em goiaba, iniciando o crescimento a partir da terceira avaliação (após oito dias da inoculação).

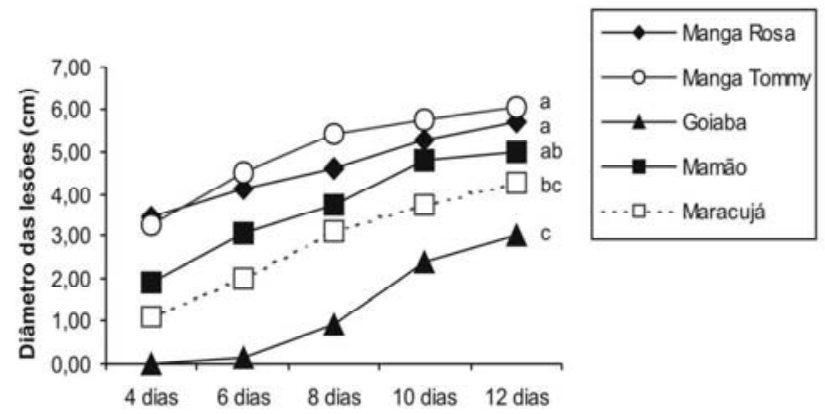

Dias após inoculação do patógeno

FIGURA 1 - Crescimento temporal do fungo Colletotrichum gloeosporioides proveniente de fruto de manga (var. Tommy Atkins) em frutos de manga var. Tommy Atkins e Rosa, maracujá, goiaba e mamão. Vitória da ConquistaBahia. 2005

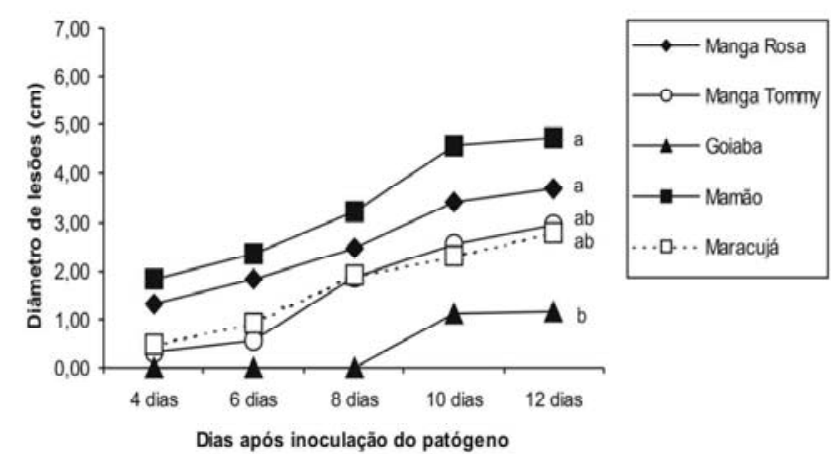

FIGURA 2 - Crescimento temporal do fungo Colletotrichum gloeosporioides proveniente de fruto de maracujá em frutos de manga var. Tommy Atkins e Rosa, maracujá, goiaba e mamão. Vitória da Conquista-Bahia. 2005.
Na Figura 3, pode-se observar que o isolado proveniente de lesões em frutos de goiaba apresentou média de crescimento de lesões inferior em seu hospedeiro de origem se comparado ao desenvolvimento das lesões nas duas variedades de manga.

Quando se inoculou o fungo proveniente de lesões em frutos de mamão nas diversas espécies frutíferas (Figuras 4), pode-se constatar que, na leitura final, o crescimento deste isolado foi superior no hospedeiro de origem, seguido por maracujá e as duas variedades de manga, sem diferirem entre si. Provavelmente essas diferenças nas intensidades de crescimento do patógeno, observadas no presente trabalho, podem estar associadas à disponibilidade de nutrientes presentes no substrato (hospedeiro) e afetarem diretamente a patogenicidade dos isolados, causando agressividade da doença, conforme constatado por Assis et al. (2001).

Nas condições em que o presente estudo foi desenvolvido, conclui-se que todos os isolados de C. gloeosporioides causaram doença em todas as frutas estudadas, demonstrando patogenicidade cruzada; a goiaba é o hospedeiro que apresenta menor crescimento de lesões resultante da inoculação de diversos isolados, inclusive da própria goiaba; as duas variedades de manga apresentam maior crescimento de lesões da antracnose.

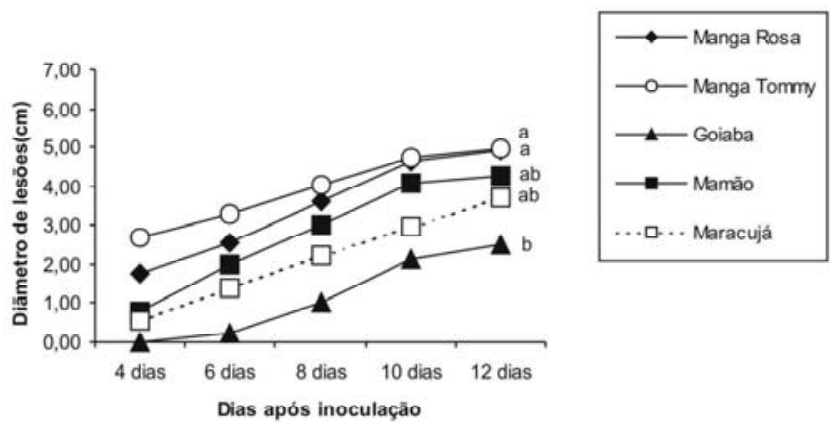

FIGURA 3 - Crescimento temporal do fungo Colletotrichum gloeosporioides proveniente de fruto de goiaba em frutos de manga, maracujá, goiaba e mamão. Vitória da Conquista-Bahia. 2005.

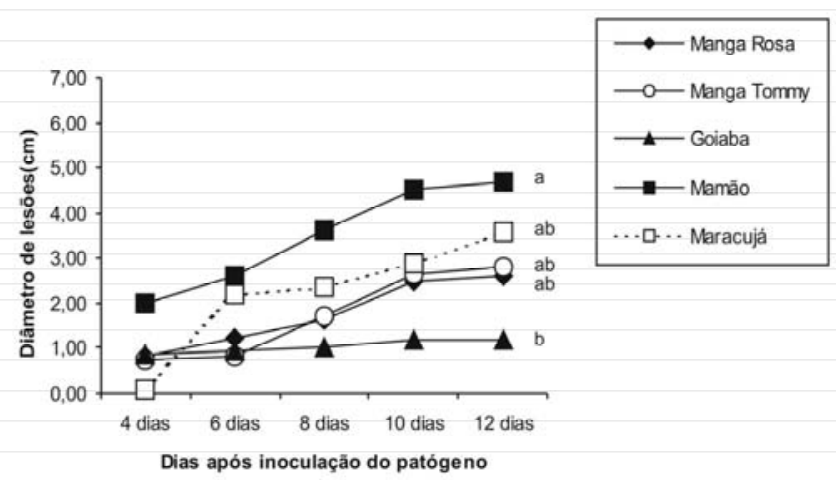

FIGURA 4 - Crescimento temporal do fungo Colletotrichum gloeosporioides proveniente de fruto de mamão em frutos de manga, maracujá, goiaba e mamão. Vitória da Conquista-Bahia. 2005.

\section{REFERÊNCIAS}

ASSIS, T. C. de; MENEZES, M.; ANDRADE,D. E. G. T.; COELHO,R. S. B.; OLIVEIRA, S. M. A. Estudo comparativo de isolados de Colletotrichum gloeosporioides quanto ao efeito da nutrição de carboidratos no crescimento, esporulação e patogenicidade em frutos de três variedades de mangueira. Summa Phytopathologica, Botucatu, v. 27, p. 208-212, 2001.

BAILEY, A. J.; JEGER, J. M. Colletotrichum: biology, pathology and 
control .Oxford: British Society for Plant Pathology, 1992. 388p.

BAILEY, J.A.; O'CONNELL, R.J.; PRING, R.J.; NASH, C. Infection strategies of Colletotrichum species. In: BAILEY, A. J.; JEGER, J. M. Colletotrichum: biology, pathology and control. Oxford: British Society for Plant Pathology, 1992. p.88-120.

GOES, A. Queda prematura de Citrus: Caracterização do agente causal, Colletotrichum gloeosporioides PENZ [SENSU ARX, 1957 e controle da doença]. 1995. Tese (Doutorado em Fitopatologia) Escola Superior de agricultura "Luiz de Queiroz", Piracicaba, SP, 1995.

LIMA FILHO, R. M.; OLIVEIRA, S. M.A.; MENEZES, M. Caracterização Enzimática e Patogenicidade Cruzada de Colletotrichum spp. Associados a Doenças de Pós-Colheita. Fitopatologia Brasileira, Brasília, v.28, n.6, p.620-625, 2003.
PERES, N. A. R.; KURAMAE, E. E.; DIAS, M. S. C.; SOUZA, N. L. de. Identification and Characterization of Colletotrichum spp. Affecting Fruit after Harvest in Brazil. Journal of Phytopathology, Berlin, v. 150, p. 128-134, 2002.

SERRA, I. M. R. de S.; SILVA, G. S. da. Caracterização Morfofisiológica de Isolados de Colletotrichum gloeosporioides Agentes de Antracnose em Frutíferas no Maranhão. Summa Phytopathologica, Botucatu, v. 30, n. 4, p. 475-480. 2004.

TAVARES, G. M. Controle químico e hidrotérmico da antracnose em frutos de mamoeiro (Carica papaya $\mathbf{L}$.) na pós-colheita. Dissertação (Mestrado em Fitopatologia) - Universidade Federal de Lavras, Lavras, MG, 2004. 55p. 